\title{
Wideband and MIMO measurements in wooded and open areas
}

\author{
Jonathan Ling, Dmitry Chizhik, Dragan Samardzija, Reinaldo Valenzuela \\ Lucent Bell Laboratories, Crawford Hill, Holmdel NJ
}

ABSTRACT- To demonstrate a real-time, mobile, peer to peer, ad-hoc networked MIMO system in a realistic tactical environment, the Defense Advanced Research Projects Agency has instituted a program called Mobile Network MIMO. The first stage of this program is to demonstrate such a system in a rural foliated environment using MIMO equipped nodes using an ad hoc network. For any particular link both nodes are naturally at ground level. Such node placement necessitates a channel measurement and modeling effort to determine quantities such as spatial correlations, delay spread as well as propagation loss.

\section{INTRODUCTION}

Use of MIMO communication techniques is of particular interest for tactical communications, where the nodes are often placed in highly scattering environments. Past measurements have found that large MIMO capacities are supported in both urban and suburban environments. To demonstrate a real-time, mobile, networked MIMO system in a realistic tactical environment, the Defense Advanced Research Projects Agency (DARPA) has instituted a program called Mobile Network MIMO (MNM) [1]. The first stage of this program is to demonstrate such a system in a rural wooded environment in Lakehurst, NJ using MIMO equipped nodes using an ad hoc network. Each node is an SUV with 8 transmit and 10 receive antennas. For any particular link both nodes are naturally at ground level. Such node placement necessitates a channel measurement and modeling effort to determine quantities such as spatial correlations, delay spread as well as propagation loss. Some of the key propagation questions are whether the channels offer enough scattering richness to benefit from MIMO systems. Also of importance is determining the delay spreads experienced in rural environments ranging from densely wooded to open field with large but sparse clutter within Line of Sight.

The capacity gain for MIMO systems is especially significant in highly scattering environments where the channel between transmit and receive antennas is uncorrelated and entries of the channel transfer matrix $\mathbf{H}$ are often assumed to be independent identically distributed (iid) complex Gaussian random variables [2]. Past measurements for cellular applications have found that large MIMO capacities are supported in both urban [3] and suburban [4] environments.

The Lakehurst Naval Air Engineering Station (NEAS) is located in the Pinelands of southern New Jersey. Of historical significance, it is the site of the 1937 Hindenburg crash. The area shown in Figure 1 is approximately $3.2 \mathrm{~km}$ by $4.8 \mathrm{~km}$. The diagonal extent, say from $2 \mathrm{~A}$ to 12 is $5 \mathrm{~km}$. Features include several very large hangers around points $\mathrm{H}, \mathrm{G}, \mathrm{V}$ and $\mathrm{X}$, open areas, runways, and mostly single story buildings. The vegetated areas consist primarily of pine trees about $10 \mathrm{~m}$ in height. There is some gentle terrain variation, as evident by the 10 foot contour intervals in Figure 1.

\footnotetext{
The research reported in this document/presentation was performed in connection with Contract No. W911NF-04-C0025 with DARPA and the U.S. Army Research Laboratory. The views and conclusions contained in this document/presentations are those of the authors and should not be interpreted as presenting the official policies or position, either expresses or implied, of the U.S. Government unless so designated by other authorized documents. Citation of manufacturer's or trade names does not constitute an official endorsement or approval of the use thereof. The U.S. Government is authorized to reproduce and distribute reprints for Government purpose not withstanding any copyright notation hereon.
} 


\section{MEASUREMENT EQUIPMENT AND PROCEDURE}

Measurements were made separately with a wideband sounder to determine delay spread and a narrowband sounder to determine spatial correlation and MIMO capacity. We first describe the wideband sounder, and measurement procedure. The narrowband multi-antenna channel sounding system was previously used in Manhattan and is described in [3].

The wideband sounder emits a length 1023 pseudo-noise sequence within $6 \mathrm{MHz}$ bandwidth centered around 2.5 $\mathrm{GHz}$. The pseudo-noise sequence has the property that the autocorrelation is -1 for all non-zero shifts. Four receivers were used to amplify, down-convert, and sample the IF waveform, and digitally down-convert to baseband. The raw data was buffered and later transferred to a PC. GPS time references are used to lock both transmit and receive local oscillators.

Measurement procedure consisted of placing the transmitter and receiver vehicles at selected points (Figure 1) to conduct wideband measurements. At each location pair, a $4 \mathrm{~W}$ signal was transmitted from a single $6 \mathrm{dBi}$ antenna and received on four widely spaced antennas, which included two $6 \mathrm{dBi}$ antennas and two $8 \mathrm{dBi}$ antennas. The receive van was driven at about $20 \mathrm{mph}$, while measurements were taken, allowing for later spatial averaging.

In post processing, the time delay of the largest peak was found by circularly convolving the received signal with the transmitted sequence. The MMSE filter was then used to extract the impulse response, positioned to capture $10 \mu \mathrm{s}$ of pre-cursors and $20 \mu \mathrm{s}$ of post-cursors. At each location, as the vehicle with the 4 receivers was driven at $20 \mathrm{mph}$, a total of $31 \times 4$ impulse responses were collected equally spread across 0.25 seconds. Assuming that the spatial correlation scale of the field is about one half wavelength $(6 \mathrm{~cm})$, about twelve independent measurements of impulse response were thus obtained. These twelve measurements were used to reduce the effect of the small scale spatial fading through incoherent (power) averaging of both the power delay profile as well as pathloss.

\section{WIDEBAND IMPULSE RESPONSE MEASUREMENTS}

Measures of delay spread such as rms delay spread, and $90^{\text {th }}$ and $95^{\text {th }}$ percentile energy support were computed from the spatially averaged power delay profiles. The time spanned by the significant measured arrivals, whose total energy was ninety percent of the energy of the measured power delay profile is defined as $90^{\text {th }}$ percentile energy support. For all measures significant arrivals are defined as having power least $5 \mathrm{~dB}$ above the average noise level. A cumulative distribution of these measures of delay spread is plotted in Figure 2. The data includes thirty nine links, where the SNR equaled or exceeded $20 \mathrm{~dB}$. It may observed that the median and $90^{\text {th }}$ percent rms delays are $.35 \mu \mathrm{s}$ and $1.7 \mu \mathrm{s}$, respectively. Our findings are slightly higher than the median rms delays for high base to mobile measurements in suburban and rural areas which were found to range from .1 to .25 $\mu$ s [5-8]. This may be caused by scattering from the four hangers which are unusually large structures, formerly housing trans-Atlantic dirigibles. Although these structures are quite unusual, delay spreads are not out of line as compared to suburban Toronto where the $90^{\text {th }}$ percent rms delay was reported as $1.2 \mu \mathrm{s}$ [8]. We conclude that delay spread for peer-to-peer ground level nodes in not significantly altered as compared to a high base positioned over the clutter. One interpretation of this is that lowering the base antenna into the clutter affects all delays equally.

The rms delay spread varied from $0.07 \mu \mathrm{s}$ in the parking lot (at about the resolution limit of the $6 \mathrm{MHz}$ sounding waveform) to $2.1 \mu \mathrm{s}$ measured on the obstructed $(23,22)$ link. Note that here and in the rest of the paper the label pair (e.g. $(23,22)$ ) corresponds to the transmitter, receiver locations, respectively. Some of the delay profiles are shown in Figures 3-5. Although it is common to model the delay profile as exponential, profiles 4 and 5 in the figures clearly do not conform to that representation. 


\section{SHORT RANGE MIMO CAPACITY}

Short-range LOS narrowband measurements were collected with van driving along both rings, as shown in Figure 1, at distances of 50 to 150 meters from each other. The vans were mostly within sight of each other for these measurements. The issue here is whether there is strong enough scattering in this LOS environment. A dual pol array was built with dimensions $3 \mathrm{~m} \times 1.5 \mathrm{~m}$, which stretches across the entire width of the van and entire rooftop length. The vertically polarized antenna have $8 \mathrm{dBi}$ vertical gain, and the horizontally polarized antenna have $9 \mathrm{dBi}$ vertical gain. For comparison with the dual polarized arrays described above, MIMO measurements were also collected using all vertically polarized arrays at both the transmitter and receiver. The antennas in each such vertically polarized array were arranged in a square grid of $1.5 \mathrm{~m} \times 1.5 \mathrm{~m}$, with about 0.5 $\mathrm{m}$ minimum antenna separation. Distribution of measured short range LOS $8 \times 10$ capacities for both dual and vertically polarized arrays is shown in Figure 6. Due to the low pathlosses, capacity is evaluated at the maximum effective SNR, here set at $23 \mathrm{~dB}$. The observed capacities are generally about $50 \%$ higher than would be expected in free space. It may be observed in this environment of low to moderate scattering and high SNR, that using the dually polarized array increases the median capacity by $46 \%$, from 30 to $44 \mathrm{bps} / \mathrm{Hz}$, over using only vertically polarized antennas.

\section{CONCLUSIONS}

An extensive campaign to characterize the wideband multi-antenna radio propagation channel between two ground based platforms was conducted in a rural area of Lakehurst, NJ. Rms delay spreads were found to have a median value of 0.4 microseconds, while the $90^{\text {th }}$ percentile rms delay spread was 1.6 microseconds. High MIMO capacity was found for short range links, albeit with a large array and dual polarization. Use of dual polarization was found to increase capacity by about $46 \%$.

\section{ACKNOWLEDGEMENT}

Many thanks are due to Cuong Tran for providing the power amplifier and assisting with logistics, and Al Sauickie for building and installing the antenna mounts. We are also grateful to other participants for helping plan the experiment as well as participating in data gathering: Dennis Romain, Charles Villa, Jesse Cohen, Baron Brown, Marc Beacken, Craig Goetjen, Alex Pidwerbetsky, and Sean Saunders. We are indebted to Peter Wolniansky for designing and constructing the narrowband channel sounder, and wideband radios. Discussions with Constantinos Papadias, and Dan Avidor have improved this work.

\section{REFERENCES}

[1] J. Freebersyser, "MNM project briefing", http://www.darpa.mil/ato/solicit/MNM/briefings/freebersyser.pdf

[2] G. J. Foschini and M. Gans, "On the limits of wireless communications in a fading environment when using multiple antennas," Wireless Personal Communications, vol. 6, March 1998.

[3] D. Chizhik, J. Ling, P. Wolniansky, R. Valenzuela, N. Costa, K. Huber, "Multiple input multiple output measurements and modeling in Manhattan", IEEE J. on Select Areas of Com., April 2003, pp. 321-331.

[4] C. Martin, J. Winters, N. Sollenberger, "Multiple-input multiple-output (MIMO) radio channel measurements", IEEE Vehicular Technology Conf., Fall 2000.

[5] L. Greenstein, V. Erceg, Y.S. Yeh, M. Clark, “A New Path-Gain/Delay-Spread Propagation Model for Digital Cellular Channels", IEEE Trans. on Veh. Tech., May 1997.

[6] P. Pajusco, "Double characterizations of power angular spectrum in macrocell environment", IEE Electronics Letters, Oct. 2003.

[7] J. Wepman, J. Hoffman, L. Loew, "Characterization of macrocellular PCS propagation channels in the 18501990 MHz Band”, Proc IEEE ICUPC, Oct 1994.

[8] E. Sousa, V. Jovanovic, C. Daigneault, "Delay spread measurements for the digital cellular channel in Toronto", IEEE Trans. on Vehicular Technology, Nov 1994 


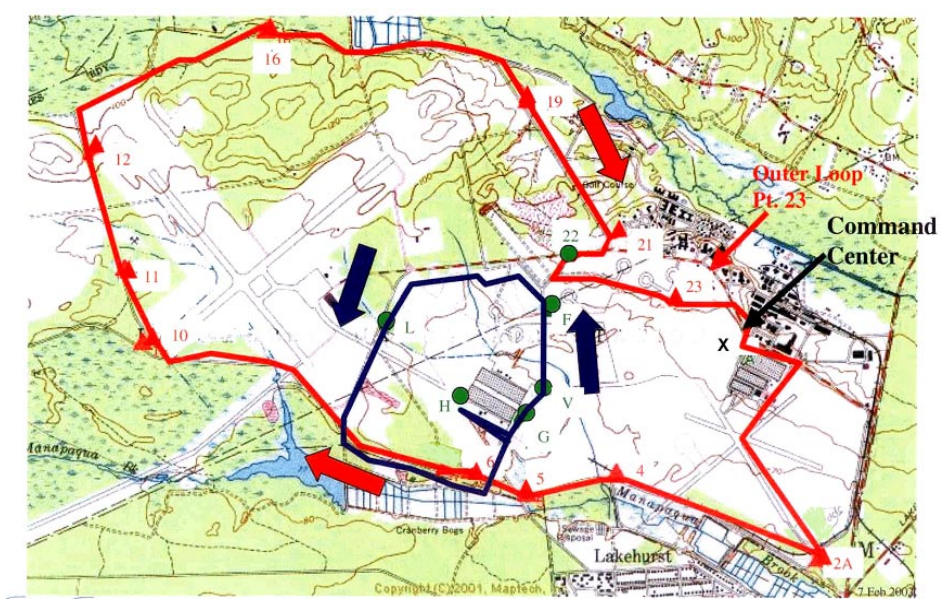

Figure 1: Map of Lakehurst NAES and the MNM demo configuration.

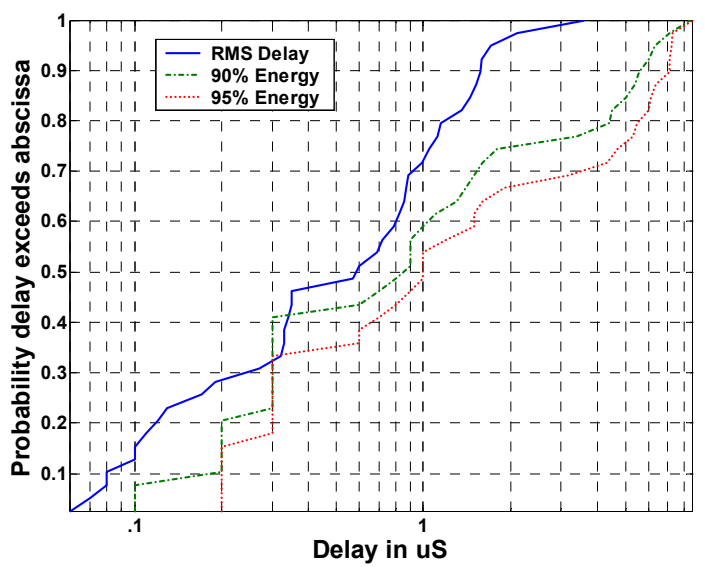

Figure 2: CDF of rms delay spread, $90^{\text {th }} \& 95^{\text {th }}$ percentile energy support

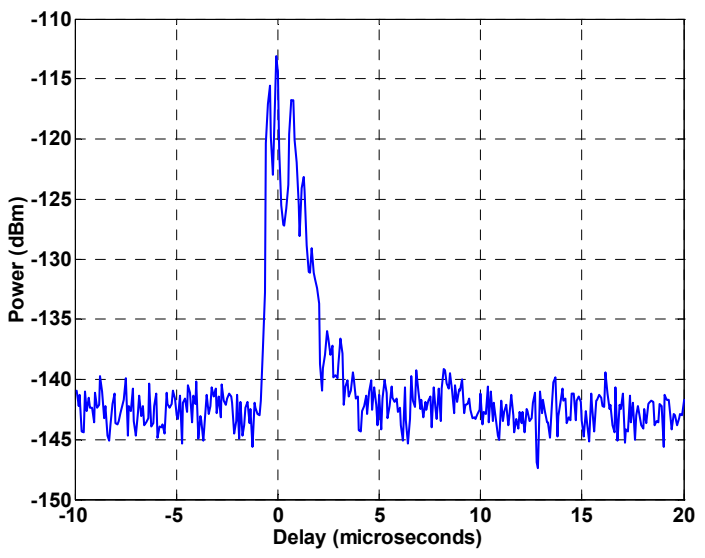

Figure 3: Delay profile from link $(\mathrm{X}, 6)$ with medium delay spread $(0.6 \mu \mathrm{S}) .145 \mathrm{~dB}$ pathloss.

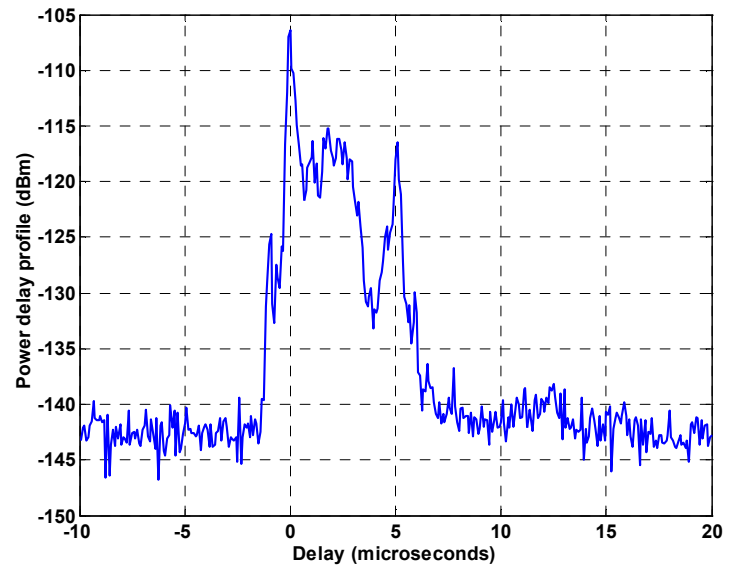

Figure 4: Delay profile from link $(\mathrm{X}, \mathrm{H})$ with high delay spread $(1.5 \mu \mathrm{S}) .138 \mathrm{~dB}$ pathloss.

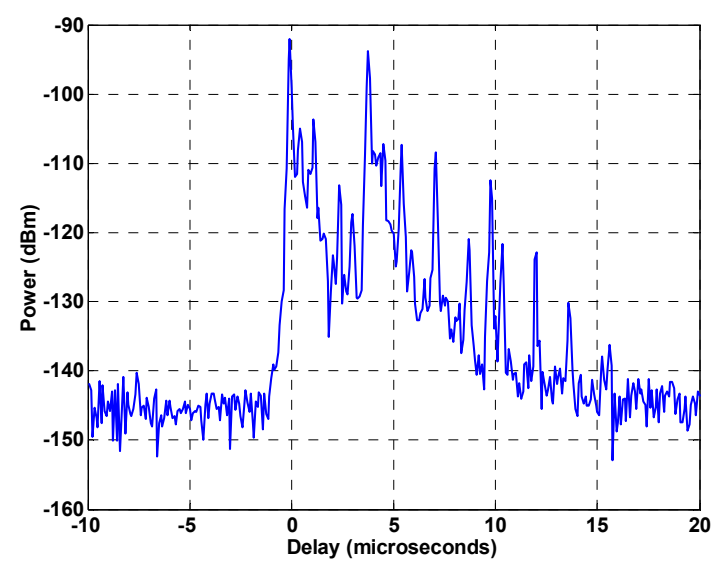

Figure 5: Delay profile from link $(23,22)$ with very high delay spread $(2.1 \mu \mathrm{s}) .125 \mathrm{~dB}$ pathloss.

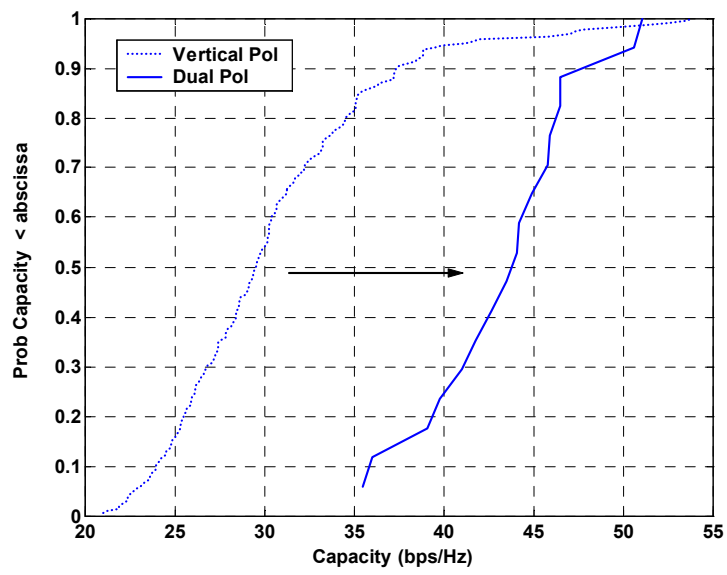

Figure 6: Distribution capacity at $23 \mathrm{~dB}$ SNR for short range LOS measurements. 